\title{
The Topology of the Coloring Complex
}

\author{
JAKOB JONSSON* \\ Department of Mathematics, KTH, SE-10044 Stockholm, Sweden
}

jakob_jonsson@yahoo.se

Received June 6, 2003; Revised August 25, 2004; Accepted September 3, 2004

\begin{abstract}
In a recent paper, E. Steingrímsson associated to each simple graph $G$ a simplicial complex $\Delta_{G}$, referred to as the coloring complex of $G$. Certain nonfaces of $\Delta_{G}$ correspond in a natural manner to proper colorings of $G$. Indeed, the $h$-vector is an affine transformation of the chromatic polynomial $\chi_{G}$ of $G$, and the reduced Euler characteristic is, up to sign, equal to $\left|\chi_{G}(-1)\right|-1$. We show that $\Delta_{G}$ is constructible and hence Cohen-Macaulay. Moreover, we introduce two subcomplexes of the coloring complex, referred to as polar coloring complexes. The $h$-vectors of these complexes are again affine transformations of $\chi_{G}$, and their Euler characteristics coincide with $\chi_{G}^{\prime}(0)$ and $-\chi_{G}^{\prime}(1)$, respectively. We show for a large class of graphs-including all connected graphs - that polar coloring complexes are constructible. Finally, the coloring complex and its polar subcomplexes being Cohen-Macaulay allows for topological interpretations of certain positivity results about the chromatic polynomial due to N. Linial and I. M. Gessel.
\end{abstract}

Keywords: topological combinatorics, constructible complex, Cohen-Macaulay complex, chromatic polynomial

\section{Introduction}

The primary goal of this paper is to analyze the topology of a certain simplicial complex $\Delta_{G}$ defined in terms of a (simple) graph $G=(V, E)$; see Section 0.1 for basic concepts and Section 1 for a formal definition of $\Delta_{G}$. The complex $\Delta_{G}$ is the coloring complex of $G$ and was introduced by Steingrímsson [17]. The faces in $\Delta_{G}$ can be interpreted as chains $\phi \neq X_{1} \varsubsetneqq X_{2} \varsubsetneqq \ldots \varsubsetneqq X_{k} \neq V$ of vertex sets with the property that the component $X_{i} \backslash X_{i-1}$ contains an edge from $G$ for at least one $i \in\{1, \ldots, k+1\}\left(X_{0}=\phi\right.$ and $\left.X_{k+1}=V\right)$.

For example, figure 1 illustrates the coloring complex $\Delta_{C_{4}}$ of the square graph $C_{4}$. $\Delta_{C_{4}}$ contains the 1 -cell $\{1,134\}$, because the component $134 \backslash 1=34$ is an edge in $C_{4}$. However, $\Delta_{C_{4}}$ does not contain the 1 -cell $\{1,124\}$, as the components $1,124 \backslash 1=24$, and $1234 \backslash 124=3$ contain no edges from $C_{4}$.

Consider the Stanley-Reisner ideal of the double cone (with apices $\phi$ and $V$ ) over the coloring complex $\Delta_{G}$. We obtain the coloring ideal $K_{G}$ of $G$ from this ideal by dividing out all monomials $x_{S} x_{T}$ such that $S \nsubseteq T$ and $T \nsubseteq S$. For each positive integer $r$, Steingrímsson [17] demonstrated that there is a bijective correspondence between proper colorings of $G$ with $r+1$ colors and monomials in $K_{G}$ of degree $r$. As a consequence, the Hilbert polynomial of $K_{G}$ is, up to a shift by one, the chromatic polynomial of $G$. This implies that the $h$-vector $\left(h_{0}, \ldots, h_{\operatorname{dim} \Delta_{G}+1}\right)$ of $\Delta_{G}$ is an affine transformation of the chromatic

* Research financed by EC's IHRP Programme, within the Research Training Network "Algebraic Combinatorics in Europe," grant HPRN-CT-2001-00272. 


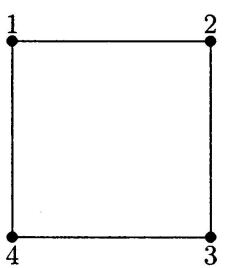

$C_{4}$

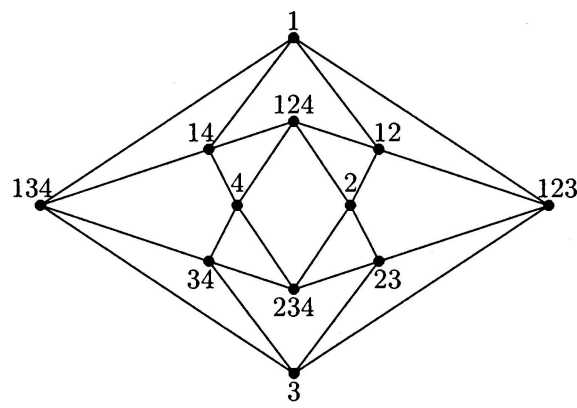

$\Delta_{C_{4}}$

Figure 1. The coloring complex of the square graph $C_{4}$.

polynomial. Specifically,

$$
\frac{\sum_{i} h_{i} t^{i}}{(1-t)^{n}}=\sum_{r \geq 0}\left((r+1)^{n}-\chi_{G}(r+1)\right) t^{r},
$$

where $\chi_{G}$ is the chromatic polynomial of $G$ and $n$ is the number of vertices in $G$. An interesting consequence is that the reduced Euler characteristic of $\Delta_{G}$ is, up to sign, equal to $\left|\chi_{G}(-1)\right|-1$. By a theorem of Stanley [14], $\left|\chi_{G}(-1)\right|$ equals the number of acyclic orientations of $G$.

- The first main result of this paper is that $\Delta_{G}$ is constructible and hence Cohen-Macaulay; see Section 1. This implies that all coefficients in the $h$-vector of $\Delta_{G}$ are nonnegative; see Stanley [16].

Greene and Zaslavsky [6] proved two theorems related to Stanley's theorem about acyclic orientations with a unique source and/or a unique sink: For every vertex $v$, the number of acyclic orientations of $G$ with the only source $v$ is equal to $\left|\chi_{G}^{\prime}(0)\right|$. Moreover, assume that $G$ contains no isolated vertices and let $v$ and $w$ be any adjacent vertices. Then the number of acyclic orientations of $G$ with the only source $v$ and the only sink $w$ is equal to $\left|\chi_{G}^{\prime}(1)\right|$. In Section 2, we introduce two subcomplexes $\Delta_{G}(v)$ and $\Delta_{G}(v, w)$ of $\Delta_{G}$, referred to as unipolar and bipolar coloring complexes, respectively. These complexes have the property that the reduced Euler characteristics are, up to sign, equal to $\left|\chi_{G}^{\prime}(0)\right|$ and $\left|\chi_{G}^{\prime}(1)\right|$, respectively—exactly the quantities in the Greene-Zaslavsky theorems.

- The second main result of this paper is that $\Delta_{G}(v)$ and $\Delta_{G}(v, w)$ are constructible and hence Cohen-Macaulay whenever the Greene-Zaslavsky theorems apply.

The unipolar and bipolar complexes being Cohen-Macaulay implies that their $h$-vectors have only nonnegative coefficients; these $h$-vectors are affine transformations of $\chi(r+1) /(r+1)$ 
and $\chi(r+1) /(r(r+1))$ similar to (1). Our positivity results are summarized in Section 3, where we also interpret analogous results by Linial [10] and Gessel [4] about the coefficients in the polynomials $(1-t)^{n+1} \cdot \sum_{r \geq 0} \chi_{G}(r+1) t^{r}$ and $(1-t)^{n-1} \cdot \sum_{r \geq 1} \chi_{G}(r+1) /(r(r+1)) \cdot t^{r}$.

\subsection{Basic concepts}

Let $G=(V, E)$ be a simple graph; $V$ is the set of vertices and $E \subseteq\left(\begin{array}{c}V \\ 2\end{array}\right)$ is the set of edges in $G$. The edge between $a$ and $b$ is denoted as $a b$. (More generally, a set $\left\{a_{1}, a_{2}, \ldots, a_{d}\right\}$ is sometimes denoted as $a_{1} a_{2} \ldots a_{d}$.) Two vertices $a$ and $b$ are adjacent in $G$ if $a b$ is an edge in $G$. An isolated vertex is a vertex not adjacent to any other vertex in $G$. Whenever the underlying vertex set $V$ is fixed, we identify $G$ with its edge set $E ; e \in G$ means that $e \in E . G$ is empty if $E=\phi$ and nonempty otherwise. Let $G-e=(V, E \backslash\{e\})$ and $G+e=(V, E \cup\{e\})$. For $W \subset V$, let $G(W)=\left(W, E \cap\left(\begin{array}{c}W \\ 2\end{array}\right)\right) ; G(W)$ is the induced subgraph of $G$ on the vertex set $W$.

For a graph $G$ and an edge $e=x y, G / e=(V / e, E / e)$ is the contraction along the edge $e$. Here, $V / e=V \backslash\{y\}$ and $E / e=(E \cup\{a x: a \neq x$ and $a y \in E\}) \cap\left(\begin{array}{c}V \backslash\{y\} \\ 2\end{array}\right)$. We refer to $y$ as the removed vertex.

For $r \geq 1,[r]$ denotes the set $\{1, \ldots, r\}$. An $r$-coloring of $G$ is a function $\gamma: V \rightarrow[r]$. A coloring $\gamma$ is proper if $\gamma(v) \neq \gamma(w)$ whenever $v w \in E$. The chromatic polynomial of $G$ is the function $\chi_{G}: \mathbb{N} \rightarrow \mathbb{N}$ with the property that $\chi_{G}(r)$ is equal to the number of proper $r$-colorings for each $r \in \mathbb{N} . \chi_{G}$ is indeed a polynomial; use (7) below. In particular, we may extend $\chi_{G}$ in a natural manner to the entire complex plane.

For a partially ordered set $P$, let $\min P$ be the set of minimal elements (sinks) in $P$. Analogously, let $\max P$ be the set of maximal elements (sources) in $P$.

A simplicial complex on a finite set $V$ is a nonempty family of subsets of $V$ closed under deletion of elements. We refer to members of a simplicial complex as faces. The dimension of a face $\sigma$ is defined as $|\sigma|-1$. The dimension of a complex $\Sigma$ is the maximal dimension of any face in $\Sigma$. A complex is pure if all maximal faces have the same dimension. For $d \geq-1$, the $d$-simplex is the simplicial complex of all subsets of a set $V$ of size $d+1$. We obtain the boundary of the $d$-simplex by removing the maximal face $V$. Note that the $(-1)$ simplex is the complex containing only the empty set. Whenever we discuss the homology of a simplicial complex, we are referring to the reduced $\mathbb{Z}$-homology.

\section{The coloring complex}

For a given nonempty graph $G$ on a vertex set $V$ of size $n$, Steingrímsson [17] defines the coloring complex $\Delta_{G}$ as follows. Let $\mathcal{F}_{V}$ be the family of all nonempty and proper subsets of $V$ (thus $\phi$ and $V$ are not contained in $\mathcal{F}_{V}$ ). A set $S$ is stable in $G$ if no edge in $G$ is contained in $S$. A (not necessarily nonempty) family

$$
\left\{X_{1}, X_{2}, \ldots, X_{k}\right\}=X_{1} X_{2} \ldots X_{k}
$$


of sets from $\mathcal{F}_{V}$, ordered from the smallest to the largest, is contained in $\Delta_{G}$ if and only if

$$
\phi=X_{0} \varsubsetneqq X_{1} \varsubsetneqq X_{2} \varsubsetneqq \cdots \varsubsetneqq X_{k} \varsubsetneqq X_{k+1}=V
$$

and at least one of the sets $Y_{i}=X_{i} \backslash X_{i-1}(1 \leq i \leq k+1)$ is not a stable set in $G$. We refer to $X_{1} X_{2} \ldots X_{k}$ as a chain and to the sets $Y_{1}, \ldots, Y_{k+1}$ as the components of the chain $X_{1} X_{2} \ldots X_{k}$. Note that the set of 0 -cells (vertices) in $\Delta_{G}$ is a proper subset of $\mathcal{F}_{V}$ if $G$ is bipartite. For example, the complex in Fig. 1 does not contain the 0 -cells 13 and 24 . The coloring complex of the graph with the single edge $e$ will be denoted as $\Delta_{e}$. While this notation is ambiguous, the underlying vertex set will always be clear from the context.

One may interpret a chain $X_{1} X_{2} \ldots X_{k}$ satisfying (2) as a coloring in which the vertices in the $i$ th component $Y_{i}$ are given color $i$ for $1 \leq i \leq k+1$. We refer to this coloring as the coloring induced by $X_{1} X_{2} \ldots X_{k}$. If $X_{1} X_{2} \ldots X_{k} \in \Delta_{G}$, then some component is non-stable, which is equivalent to saying that the induced coloring is not proper.

$\Delta_{G}$ is easily seen to be pure of dimension $n-3$. Indeed, one may describe the maximal faces of $\Delta_{G}$ as follows. A labeling of $V$ is a bijection from $V$ to $[n]=\{1, \ldots, n\}$. For a labeling $\omega$, define $X_{\omega, i}=\omega^{-1}([i])$ and

$$
\mathcal{X}_{\omega}=X_{\omega, 1} X_{\omega, 2} \ldots X_{\omega, n-1}
$$

A chain $\mathcal{X}$ is a maximal face if and only if there is a labeling $\omega$ and an integer $i \in[n-1]$ such that $\mathcal{X}=\mathcal{X}_{\omega, i}$, where

$$
\mathcal{X}_{\omega, i}=\mathcal{X}_{\omega} \backslash X_{\omega, i}=X_{\omega, 1} \ldots \hat{X}_{\omega, i} \ldots X_{\omega, n-1}
$$

(the hat denotes deletion), and such that the vertices $\omega^{-1}(i)$ and $\omega^{-1}(i+1)$ are adjacent in $G$. Namely, such a chain induces a coloring of $G$ with the property that the two adjacent vertices $\omega^{-1}(i)$ and $\omega^{-1}(i+1)$ are given the same color.

There is a natural ring-theoretic interpretation of the above concepts; see Steingrímsson [17] for a more detailed discussion. Let $F$ be a field and define $A=A_{V}=F\left[x_{S}: S \subseteq V\right]$, $I=I_{V}=\left\{x_{S} x_{T}: S \nsubseteq T, T \nsubseteq S\right\}$, and $R=R_{V}=A / I$. For a graph $G$, consider the set of all monomials $x_{X_{1}}^{e_{1}} x_{X_{2}}^{e_{2}} \cdots x_{X_{k}}^{e_{k}}$ such that $X_{1} X_{2} \ldots X_{k}$ satisfies (2) except possibly at the endpoints; thus we allow $X_{1}$ to be equal to $\phi$ and $X_{k}$ to be equal to $V$. Let $K_{G}$ be generated by exactly those monomials $x_{X_{1}}^{e_{1}} x_{X_{2}}^{e_{2}} \cdots x_{X_{k}}^{e_{k}}\left(e_{i}>0\right)$ with the property that all components of $X_{1} X_{2} \ldots X_{k}$ are stable in $G$. Then $R / K_{G}$ is the Stanley-Reisner ring of the double cone over $\Delta_{G}$, where the two added apices correspond to the sets $\phi$ and $V$.

Each monomial of degree $d$ in $R=R_{V}$ corresponds to a $(d+1)$-coloring of the vertex set $V$. Namely, let $x_{X_{1}}^{e_{1}} x_{X_{2}}^{e_{2}} \cdots x_{X_{k}}^{e_{k}}$ be a monomial in $R$ and let $Y_{1}, \ldots, Y_{k+1}$ be the components of the chain $X_{1} \ldots X_{k}$. We obtain a coloring by giving all vertices in the set $Y_{i}$ the color $\sum_{j<i} e_{j}+1$. In fact, Steingrímsson [17] showed that this gives a bijection between monomials of $R$ and colorings of $V$. Moreover, for each graph $G$, there is a bijection between monomials of $K_{G}$ and proper colorings of $G$. 


\subsection{The homotopy type of the coloring complex}

As $\Delta_{G}$ is a pure complex, a natural question to ask is whether $\Delta_{G}$ is Cohen-Macaulay. The object of this section is to verify that this is indeed the case.

Definition 1.1 The class of constructible simplicial complexes is defined recursively as follows.

1. Every simplex (including the $(-1)$-simplex $\{\phi\}$ ) is constructible.

2. If $\Delta_{1}$ and $\Delta_{2}$ are constructible complexes of dimension $d$ and $\Delta_{1} \cap \Delta_{2}$ is a constructible complex of dimension $d-1$, then $\Delta_{1} \cup \Delta_{2}$ is constructible.

The concept of constructible complexes was introduced by Hochster [8]. Every shellable complex is constructible, but the converse is not always true; see Björner [1].

Definition 1.2 A simplicial complex $\Delta$ is homotopy-Cohen-Macaulay (abbreviated homotopy-C $\mathrm{M}$ ) if every link of $\Delta$ (including $\Delta$ itself) is homotopy equivalent to a wedge of spheres in top dimension. This means that $\operatorname{link}_{\Delta}(\sigma)$ is $\left(\operatorname{dim~link}_{\Delta}(\sigma)-1\right)$-connected for each $\sigma \in \Delta$; see Björner [1]. For a ring $R, \Delta$ is Cohen-Macaulay over $R$ (denoted as $C M / R)$ if $\tilde{H}_{i}\left(\operatorname{link}_{\Delta}(\sigma) ; R\right)=0$ whenever $i<\operatorname{dim}_{\operatorname{link}_{\Delta}}(\sigma)$ for each $\sigma \in \Delta$ (including $\sigma=\phi)$.

See Reisner [13] for the ring-theoretic motivation of Definition 1.2. Any constructible complex is also homotopy- $C M$ and any homotopy- $C M$ complex is also $C M / \mathbb{Z}$ (and $C M / k$ for any field $k$ ), but the converses do not hold in general; see Björner [1] for more information.

Lemma 1.3 If $G$ is a graph with at least two edges, then $\Delta_{G-e} \cap \Delta_{e}$ and $\Delta_{G / e}$ are isomorphic for any edge e in $G$.

Proof: Let $e=x y$ and let $y$ be the removed vertex in $G / e$. For a chain $\mathcal{X} \in \Delta_{e}$ with components $Y_{1}, \ldots, Y_{k+1}$, let $Y_{i}$ be the component containing $x$ and $y$; they must be in the same component. Define $\varphi(\mathcal{X})$ to be the chain with components $Y_{1}, \ldots, Y_{i} \backslash\{y\}, \ldots, Y_{k+1}$; all components but $Y_{i}$ remain unchanged. This clearly gives an isomorphism from $\Delta_{e}$ to the complex $\Sigma_{V \backslash\{y\}}$ of all possible chains of the form (2) on the vertex set $V \backslash\{y\}$. Namely, we may easily reconstruct a chain $\mathcal{X}=\varphi^{-1}\left(\mathcal{X}^{\prime}\right) \in \Delta_{e}$ from a chain $\mathcal{X}^{\prime} \in \Sigma_{V \backslash\{y\}}$ by adding $y$ to the component containing $x$. As a consequence, we need only prove for each $\mathcal{X} \in \Delta_{e}$ that $\mathcal{X} \in \Delta_{G-e}$ if and only if $\varphi(\mathcal{X}) \in \Delta_{G / e}$.

Let $\mathcal{X} \in \Delta_{e}$ and let $Y_{i}$ be the component containing $x$ and $y$. Clearly, each of the other components is stable in $G-e$ if and only if it is stable in $G / e$. Moreover, the same is true for $Y_{i}$. Namely, for each $z \in Y_{i} \backslash\{x, y\}, x z$ is an edge in $G / e$ if and only if at least one of $x z$ and $y z$ is an edge in $G-e$. It follows that $\mathcal{X} \in \Delta_{G-e}$ if and only if $\varphi(\mathcal{X}) \in \Delta_{G / e}$.

Theorem 1.4 For any nonempty graph $G$ on $n$ vertices, $\Delta_{G}$ is constructible. As a consequence, $\Delta_{G}$ is homotopy-CM. In particular, $\Delta_{G}$ is homotopy equivalent to a wedge of spheres of dimension $n-3$. 
Remark. The homotopy type of $\Delta_{G}$ has been determined earlier by Herzog et al. [7]; see the remark after Corollary 1.8 .

Proof: We use induction on the number of vertices and the number of edges in $G$. First, suppose $G$ has only one edge $e$. Then $\Delta_{G}=\Delta_{e}$ is isomorphic to the first barycentric subdivision of the boundary of an $(n-2)$-simplex; see the proof of Lemma 1.3. This complex is shellable (see Björner and Wachs [2]), which implies that $\Delta_{e}$ is constructible.

Now, consider a graph $G$ with at least two edges. By induction, we may assume that we have already proved that all coloring complexes of nonempty graphs with fewer edges than $G$ are constructible. Let $e$ be an arbitrary edge in $G$. It is clear that

$$
\Delta_{G}=\Delta_{G-e} \cup \Delta_{e}
$$

Note that each of $\Delta_{G-e}$ and $\Delta_{e}$ is pure of dimension $n-3$. By the induction hypothesis, both complexes are constructible.

It remains to prove that $\Delta_{G-e} \cap \Delta_{e}$ is constructible of dimension $n-4$. Now, Lemma 1.3 implies that $\Delta_{G-e} \cap \Delta_{e}$ is isomorphic to $\Delta_{G / e}$. Since the number of vertices in $G / e$ is $n-1$ and the number of edges is at least one, we are done by induction.

Theorem 1.5 If $G$ is a graph with at least two edges and $e$ is an edge in $G$, then the reduced Euler characteristic $\tilde{\chi}\left(\Delta_{G}\right)$ of $\Delta_{G}$ satisfies

$$
\tilde{\chi}\left(\Delta_{G}\right)=\tilde{\chi}\left(\Delta_{G-e}\right)-\tilde{\chi}\left(\Delta_{G / e}\right)+\tilde{\chi}\left(\Delta_{e}\right) .
$$

In particular, any graph $G$ satisfies

$$
\tilde{\chi}\left(\Delta_{G}\right)=-\chi_{G}(-1)+(-1)^{n}
$$

where $\chi_{G}$ is the chromatic polynomial of $G$. Thus $\Delta_{G}$ is homotopy equivalent to a wedge of $\left|\chi_{G}(-1)\right|-1$ spheres of dimension $n-3$.

Remark (6) was first proved by Steingrímsson [17].

Proof: The identity (5) is an immediate consequence of the identity

$$
\Delta_{G}=\left(\Delta_{G-e} \backslash\left(\Delta_{G-e} \cap \Delta_{e}\right)\right) \cup \Delta_{e}
$$

and Lemma 1.3; the union is clearly disjoint. Combining this identity with the well-known recursive property

$$
\chi_{G}(t)=\chi_{G-e}(t)-\chi_{G / e}(t)
$$

of chromatic polynomials, we obtain via induction that

$$
\begin{aligned}
\tilde{\chi}\left(\Delta_{G}\right) & =-\chi_{G-e}(-1)+(-1)^{n}+\chi_{G / e}(-1)-(-1)^{n-1}-\chi_{e}(-1)+(-1)^{n} \\
& =-\chi_{G}(-1)+3 \cdot(-1)^{n}-\chi_{e}(-1)=-\chi_{G}(-1)+(-1)^{n} .
\end{aligned}
$$


The base case is that $G$ consists of a single edge $e$. Yet, we already know that $\Delta_{e}$ is the first barycentric subdivision of the boundary of an $(n-2)$-simplex and hence homeomorphic to a sphere of dimension $(n-3)$; thus

$$
\tilde{\chi}\left(\Delta_{e}\right)=(-1)^{n-3}=-(-1)^{n}=-\chi_{e}(-1)+(-1)^{n} .
$$

For the last statement in the theorem, note that the sign of $\chi_{G}(-1)$ is always $(-1)^{n}$.

For a simplicial complex $\Delta$ of dimension $d-1$, define $f_{i}$ as the number of faces of dimension $i$ in $\Delta ;\left(f_{0}, \ldots, f_{d-1}\right)$ is the $f$-vector of $\Delta$. Define the $h$-vector $\left(h_{0}, \ldots, h_{d}\right)$ of $\Delta$ by the formula

$$
\sum_{i=0}^{d} f_{i-1} t^{i}(1-t)^{d-i}=\sum_{i=0}^{d} h_{i} t^{i}
$$

$f_{-1}=1$. Let $h(\Delta, t)=\sum_{i=0}^{d} h_{i} t^{i}$ and $f(\Delta, u)=\sum_{i=0}^{d} f_{i-1} u^{i}$. With $u=t /(1-t)$, it is clear that

$$
\frac{h(\Delta, t)}{(1-t)^{d}}=f(\Delta, u)
$$

As Steingrímsson [17] showed, (6) is a consequence of the following result.

Theorem 1.6 (Steingrímsson [17]) Let $u=t /(1-t)$. For any nonempty graph $G$,

$$
\frac{h\left(\Delta_{G}, t\right)}{(1-t)^{n}}=f\left(\Delta_{G}, u\right) \cdot(1+u)^{2}=\sum_{r \geq 0} T_{G}(r+1) t^{r},
$$

where $n$ is the number of vertices in $G$ and $T_{G}(r)=r^{n}-\chi_{G}(r)$.

Remark To be precise, Steingrímsson proved Theorem 1.6 for the double cone over the complex $\Delta_{G}$, meaning that chains are allowed to contain the empty set $\phi$ and the full set $V$. Note that the polynomial $f\left(\Delta_{G}, u\right) \cdot(1+u)^{2}$ in (8) corresponds to the $f$-vector of this double cone.

We obtain an orientation of $G$ by directing each edge $a b$ in $G$, either from $a$ to $b$ or from $b$ to $a$. One may view this as an asymmetric relation $\prec$ on the pairs of adjacent vertices $a$ and $b$ in $G$; exactly one of $a \prec b$ and $b \prec a$ holds. The orientation is acyclic if the transitive closure of $\prec$ gives a partial order $P$ on $V$. We will identify the acyclic orientation with this partial order. (This is slightly unconventional; most authors identify the acyclic orientation with the underlying directed graph.) Let $\mathcal{P}_{G}$ denote the set of acyclic orientations of $G$ and let $A_{G}=\left|\mathcal{P}_{G}\right|$ denote the number of acyclic orientations of $G$.

Theorem 1.7 (Stanley [14]) For any graph $G, A_{G}=(-1)^{n} \cdot \chi_{G}(-1)$, where $n$ is the number of vertices in $G$. 
As Steingrímsson [17] observed, Theorem 1.7, combined with (6), implies that

$$
\tilde{\chi}\left(\Delta_{G}\right)=(-1)^{n-1} \cdot\left(A_{G}-1\right) .
$$

Summarizing, we obtain the following corollary:

Corollary $1.8 \Delta_{G}$ is homotopy equivalent to a wedge of $A_{G}-1$ spheres of dimension $n-3$.

Remark Herzog, Reiner, and Welker [7] were the first to establish Corollary 1.8; in their paper, combine Lemmas 3.2 and 6.3 as described in the proof of Theorem 4.2. They denoted the coloring complex as $\Delta_{m, J}$, where $m$ and $J$ correspond to the vertex set and the edge set, respectively, of $G$. Their proof was based on the "graphic" hyperplane arrangements used by Greene and Zaslavsky in an alternative proof [6] of Theorem 1.7.

Before proceeding, let us state some well-known facts about barycentric subdivisions. The $n$th Eulerian polynomial $E_{n}(t)$ is defined by

$$
\frac{E_{n}(t)}{(1-t)^{n+1}}=\sum_{r \geq 1} r^{n} t^{r}
$$

Proposition 1.9 (Folklore) The (cone over the) first barycentric subdivision of the (boundary of the) $n$-simplex has h-vector $E_{n+1}(t) / t$.

Note that the first barycentric subdivision of the $n$-simplex coincides with the cone over the first barycentric subdivision of the boundary of the $n$-simplex.

\section{Polar coloring complexes}

Greene and Zaslavsky [6] proved the following results analogous to Stanley's Theorem 1.7; see the work of Gebhard and Sagan [5] for alternative proofs.

Theorem 2.1 (Greene-Zaslavsky [6]) Let $G$ be a graph and let $v$ be a vertex in $G$. The number of acyclic orientations $P$ of $G$ such that $\max P=\{v\}$ is equal to $(-1)^{n-1} \cdot \chi_{G}^{\prime}(0)$; this is the absolute value of the linear coefficient in $\chi_{G}$.

Theorem 2.2 (Greene-Zaslavsky [6]) Let $G$ be a graph, let $S$ be the set of isolated vertices in $G$, and let $v$ and $w$ be adjacent vertices in $G$. The number of acyclic orientations $P$ of $G(V \backslash S)$ with $\max P=\{v\}$ and $\min P=\{w\}$ is equal to $(-1)^{n-|S|} \cdot \chi_{G}^{\prime}(1)$.

Let $G$ be a graph. For a vertex $v$ in $G$, let $\Delta_{G}(v)$ be the induced subcomplex of $\Delta_{G}$ obtained by removing all 0 -cells $X$ containing $v$. We refer to $\Delta_{G}(v)$ as the unipolar coloring complex 


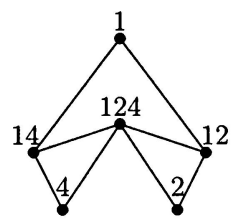

$\Delta_{C_{4}}(3)$

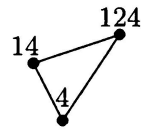

$\Delta_{C_{4}}(3,4)$

Figure 2. Unipolar and bipolar subcomplexes of $\Delta_{C_{4}}$; compare to Fig. 1.

of $G$ (with source $v$ ). For any two vertices $v$ and $w$ in $G$, let $\Delta_{G}(v, w)$ be the induced subcomplex of $\Delta_{G}(v)$ obtained by removing all 0 -cells $X$ not containing $w$. We refer to this complex as the bipolar coloring complex of $G$ (with source $v$ and sink $w$-one may view these vertices as "poles"). See figure 2 for examples. Note that $\Delta_{G+v w}(v, w)$ and $\Delta_{G-v w}(v, w)$ coincide except when $G-v w$ is empty. We refer to unipolar and bipolar complexes jointly as polar complexes. A bipolar orientation is an acyclic orientation with a unique source and a unique sink.

Lemma 2.3 For any nonempty graph $G$ and any edge $e \in G$,

$$
\begin{aligned}
\Delta_{G}(v) & =\Delta_{G-e}(v) \cup \Delta_{e}(v) ; \\
\Delta_{G}(v, w) & =\Delta_{G-e}(v, w) \cup \Delta_{\{e, v w\}}(v, w) ;
\end{aligned}
$$

$\Delta_{\{e, v w\}}(v, w)$ is the bipolar coloring complex of the graph $(V,\{e, v w\})$.

Proof: This is immediate from the fact that these complexes are induced subcomplexes of $\Delta_{G}, \Delta_{G-e}$, and $\Delta_{e}\left(\Delta_{\{e, v w\}}(v, w)=\Delta_{e}(v, w)\right)$; compare to (4).

A simple induction argument yields that $\Delta_{G}(v)$ and $\Delta_{G}(v, w)$ are pure of dimension $n-3$; we consider the base steps $G=(V, e)$ and $G=(V,\{e, v w\})$ in the proofs of Theorems 2.5 and 2.6 below.

Lemma 2.4 For any nonempty graph $G$ and any edge $e \in G$,

$$
\begin{aligned}
\Delta_{G / e}(v) & \cong \Delta_{G-e}(v) \cap \Delta_{e}(v) ; \\
\Delta_{G / e}(v, w) & \cong \Delta_{G-e}(v, w) \cap \Delta_{\{e, v w\}}(v, w)
\end{aligned}
$$

$(\cong$ denotes isomorphism); $e \neq v w$ in $(13)$.

Remark If $e=v x$ in (12) or if $e=v x$ or $e=w x$ in (13), then $x$ is the removed vertex in $G / e$.

Proof: (12) follows immediately from the proof of Lemma 1.3; the transformation $\varphi$ preserves the index $i$ of the component $Y_{i}$ containing $v$. The same is true for (13). Namely, 
$\Delta_{\{e, v w\}}(v, w)=\Delta_{e}(v, w)$, and $\varphi$ preserves the index $j$ of the component $Y_{j}$ containing $w$.

Before analyzing the topology of $\Delta_{G}(v)$ and $\Delta_{G}(v, w)$, we will prove the following results corresponding to Theorem 1.6; an alternative ring-theoretic approach to proving these results is given at the end of this section.

Theorem 2.5 Let $u=t /(1-t)$. For any nonempty graph $G$ and any vertex $v$ in $G$, the unipolar coloring complex $\Delta_{G}(v)$ satisfies

$$
\frac{h\left(\Delta_{G}(v), t\right)}{(1-t)^{n-1}}=f\left(\Delta_{G}(v), u\right) \cdot(1+u)=\sum_{r \geq 0} \frac{T_{G}(r+1)}{r+1} \cdot t^{r}
$$

where $n$ is the number of vertices in $G$ and $T_{G}(r)=r^{n}-\chi_{G}(r)$. Moreover, the reduced Euler characteristic of $\Delta_{G}(v)$ is $\chi_{G}^{\prime}(0)$.

Proof: If $G$ is a graph on $n$ vertices with one edge, then $\Delta_{G}(v)$ is the first barycentric subdivision of an $(n-3)$-simplex, which by Proposition 1.9 has $h$-polynomial $E_{n-2}(t) / t$. Since $T_{G}(r) / r=r^{n-2}$, (14) follows.

Now, assume that $G$ is a graph with at least two edges. Let $e$ be any edge in $G$. By induction, we may assume that

$$
f\left(\Delta_{H}(v), u\right) \cdot(1+u)=\sum_{r \geq 0} \frac{T_{H}(r+1)}{r+1} \cdot t^{r}
$$

for $H \in\{G-e, e, G / e\}$. (10) and (12) yield that $f\left(\Delta_{G}(v), u\right) \cdot(1+u)$ is equal to

$$
\begin{aligned}
& \left(f\left(\Delta_{G-e}(v), u\right)+f\left(\Delta_{e}(v), u\right)-f\left(\Delta_{G / e}(v), u\right)\right) \cdot(1+u) \\
& \quad=\sum_{r \geq 0} \frac{T_{G-e}(r+1)}{r+1} \cdot t^{r}+\sum_{r \geq 0} \frac{T_{e}(r+1)}{r+1} \cdot t^{r}-\sum_{r \geq 0} \frac{T_{G / e}(r+1)}{r+1} \cdot t^{r} \\
& \quad=\sum_{r \geq 0} \frac{T_{G}(r+1)}{r+1} \cdot t^{r} .
\end{aligned}
$$

The last equality is a consequence of (7) and the fact that $\chi_{e}(r)=r^{n}-r^{n-1}$. The reduced Euler characteristic is determined in the same manner.

Theorem 2.6 Let $u=t /(1-t)$. For any graph $G$ with at least two edges and any adjacent vertices $v$ and $w$, the bipolar coloring complex $\Delta_{G}(v, w)$ satisfies

$$
\frac{h\left(\Delta_{G}(v, w), t\right)}{(1-t)^{n-2}}=f\left(\Delta_{G}(v, w), u\right)=\sum_{r \geq 1} \frac{U_{G}(r+1)}{r(r+1)} \cdot t^{r}+1,
$$

where $U_{G}(r)=T_{G}(r)-r^{n-1}=r^{n}-r^{n-1}-\chi_{G}(r)$. Moreover, the reduced Euler characteristic of $\Delta_{G}(v, w)$ is $-\chi_{G}^{\prime}(1)$. 
Proof: If $G$ is a graph on $n$ vertices with two edges (one of them being $v w$ ), then $\Delta_{G}(v, w)$ is a cone over the first barycentric subdivision of an $(n-4)$-simplex, which means that $\Delta_{G}(v, w)$ has $h$-polynomial $E_{n-3}(t) / t$; see Proposition 1.9. Since $\chi_{G}(r)=(r-1)^{2} r^{n-2}$, it is clear that $U_{G}(r+1) /(r(r+1))=(r+1)^{n-3}$. It follows that

$$
\frac{h\left(\Delta_{G}(v, w), t\right)}{(1-t)^{n-2}}=\frac{E_{n-3}(t) / t}{(1-t)^{n-2}}=\sum_{r \geq 0}(r+1)^{n-3} t^{r}=\sum_{r \geq 1}(r+1)^{n-3} t^{r}+1,
$$

which implies (15).

If $G$ is a triangle graph on $n$ vertices containing the three edges $v w, v x$, and $w x$ for some $x$ and no other edge, then $\Delta_{G}(v, w)=\Delta_{\{v w, v x\}} \cup \Delta_{\{v w, w x\}}$; note that we just determined the $h$-vector of the complexes in the right-hand side. Since $\Delta_{\{v w, v x\}} \cap \Delta_{\{v w, w x\}}$ is the (-1)simplex, the $h$-polynomial of $\Delta_{G}$ satisfies

$$
\begin{aligned}
\frac{h\left(\Delta_{G}(v, w), t\right)}{(1-t)^{n-2}} & =\frac{2 E_{n-3}(t) / t}{(1-t)^{n-2}}-1=\sum_{r \geq 0} 2(r+1)^{n-3} t^{r}-1 \\
& =\sum_{r \geq 1} 2(r+1)^{n-3} t^{r}+1
\end{aligned}
$$

again (15) follows, because $U_{G}(r+1) /(r(r+1))=2(r+1)^{n-3}$.

Now, assume that $G$ is a graph with at least three edges and $G$ is not the triangle graph. Let $e$ be any edge in $G-v w$. Since $G$ is not the triangle graph, $G / e$ contains at least two edges. By induction, we may hence assume that

$$
f\left(\Delta_{H}(v), u\right)=\sum_{r \geq 1} \frac{U_{H}(r+1)}{r(r+1)} \cdot t^{r}+1
$$

for $H \in\{G-e,\{v w, e\}, G / e\}$. (11) and (13) yield that $f\left(\Delta_{G}(v), u\right)$ is equal to

$$
\begin{aligned}
f & \left(\Delta_{G-e}(v, w), u\right)+f\left(\Delta_{\{e, v w\}}(v, w), u\right)-f\left(\Delta_{G / e}(v, w), u\right) \\
& =\sum_{r \geq 1} \frac{U_{G-e}(r+1)}{r(r+1)} \cdot t^{r}+\sum_{r \geq 1} \frac{U_{\{e, v w\}}(r+1)}{r(r+1)} \cdot t^{r}-\sum_{r \geq 1} \frac{U_{G / e}(r+1)}{r(r+1)} \cdot t^{r}+1 \\
& =\sum_{r \geq 1} \frac{U_{G}(r+1)}{r(r+1)} \cdot t^{r}+1 .
\end{aligned}
$$

The last equality is a consequence of (7) and the fact that $\chi_{\{e, v w\}}(r)=\left(r^{n}-r^{n-1}\right)-\left(r^{n-1}-\right.$ $\left.r^{n-2}\right)$. The reduced Euler characteristic is determined in the same manner.

Remark An alternative approach to proving Theorems 2.5 and 2.6 would be to use ring theory; recall notation from Section 1. Let $J(v)=\left\{x_{I}: v \in I\right\}$ and define $R(v)=R / J(v)$ and $K_{G}(v)=K_{G} / J(v) ; K_{G}(v)$ is an ideal in $R(v)$. It is easy to see that $R(v) / K_{G}(v)$ coincides with the Stanley-Reisner ring of the cone over $\Delta_{G}(v)$ with apex $\phi$. Namely, we 
divide out exactly those monomials in $R / K_{G}$ that contain a factor $x_{I}$ such that $v \in I$. Such sets $I$ are precisely the ones that we removed from $\Delta_{G}$ to obtain $\Delta_{G}(v)$.

It is clear that the Hilbert polynomial of $R(v)$ is $(r+1)^{n-1}$, the ring being the StanleyReisner ring of a cone over the first barycentric subdivision of an $(n-2)$-simplex. Moreover, by the discussion at the end of Section 1, the monomials of degree $d$ in $K_{G}(v)$ correspond precisely to proper $(d+1)$-colorings of $G$ such that $v$ is given color $d+1$. As a consequence, the Hilbert polynomial of $K_{G}(v)$ is $\chi_{G}(r+1) /(r+1)$. (14) now follows quite easily (note that we have left out some technical details).

Similar interpretations exist for $\Delta_{G}(v, w) ; J(v, w)$ is the set of monomials $x_{I}$ such that either $v \in I$ or $w \notin I$. This time, $R(v, w)=R / J(v, w)$ is the Stanley-Reisner ring of the first barycentric subdivision of an $(n-3)$-simplex, which means that the Hilbert polynomial is $(r+1)^{n-2}$. Moreover, $K_{G}(v, w)=K_{G} / J(v, w)$ contains $d$-degree monomials corresponding to $(d+1)$-colorings in which $w$ is given color 1 and $v$ is given color $d+1$; hence the Hilbert polynomial is $\chi_{G}(r+1) /(r(r+1))$.

\subsection{The homotopy type of polar coloring complexes}

We now proceed with the analogues of Theorem 1.4; we use the same proof techniques as in Section 1.1. It is conceivable that one may apply hyperplane arrangements as in the proof by Herzog et al. [7] of Corollary 1.8; compare to Greene and Zaslavsky [6]. We do not know whether such an approach would give any information about Cohen-Macaulayness.

Using our approach, it turns out that the situation is substantially more complicated for the smaller bipolar complex $\Delta_{G}(v, w)$ than for the larger unipolar complex $\Delta_{G}(v)$.

Theorem 2.7 For any nonempty graph $G$ on $n$ vertices and any vertex $v$ in $G$, the unipolar coloring complex $\Delta_{G}(v)$ is a constructible complex. As a consequence, $\Delta_{G}(v)$ is homotopy$C M$. In particular, $\Delta_{G}(v)$ is homotopy equivalent to a wedge of spheres of dimension $n-3$ with one sphere for each acyclic orientation $P$ of $G$ such that $\max P=\{v\}$.

Proof: We use induction on the number of vertices and edges in $G$. We have already concluded that $\Delta_{e}(v)$ is the first barycentric subdivision of the $(n-3)$-simplex and hence shellable. If $G$ has at least two edges and $e \in G$, then by induction we may assume that $\Delta_{G-e}(v), \Delta_{e}(v)$, and $\Delta_{G / e}(v)$ are constructible. Lemmas 2.3 and 2.4 immediately imply that $\Delta_{G}(v)$ is constructible. The last statement in the Theorem is a consequence of the Greene-Zaslavsky Theorem 2.1.

The bipolar coloring complex $\Delta_{G}(v, w)$ is not always constructible. For example, if $G$ is a disconnected graph with only three edges forming a triangle, then $\Delta_{G}(v, w)$ is disconnected. To describe the property needed for a bipolar coloring complex to be constructible, some notation is needed. A graph $G$ is 2-connected if, for any vertex $v$ in $G$, the induced subgraph $G(V \backslash\{v\})$ is connected. A vertex $x$ such that $G(V \backslash\{x\})$ is disconnected is a cutpoint in $G$. For the purposes of this paper, the graph with two vertices and one edge is 2-connected, whereas the singleton graph on one vertex is not. This convention might be nonstandard but aligns quite well with the following well-known result. 
Theorem 2.8 (Lempel et al. [9]) Let $G$ be a graph and let $v$ and $w$ be adjacent vertices in $G$. Then $G$ admits a bipolar acyclic orientation $P$ with $\max P=\{v\}$ and $\min P=\{w\}$ if and only if $G$ is 2-connected.

Let $G=(V, E)$ be a graph and let $S$ be the set of isolated vertices in $G$. It is well-known (see Lovász [11]) that each edge $e$ in $G$ is contained in a unique maximal set $B_{e}$ with the property that the induced subgraph $G\left(B_{e}\right)$ is 2-connected. We refer to $B_{e}$ as a 2-connected component. Note that the 2-connected components of $G$ induce a partition of the edge set E.

Say that $G$ is pleasant if at least one of the following two properties holds.

1. $G$ is connected.

2. $G$ has at least two 2-connected components.

Accordingly, a graph is unpleasant if it contains isolated vertices and if the graph obtained by removing all isolated vertices is 2-connected. (In a wider context, there is of course nothing unpleasant about such graphs; the terminology is intended only for the purposes of this paper.) The following two theorems demonstrate that a graph is pleasant if and only if the bipolar coloring complex $\Delta_{G}(v, w)$ is constructible; the choice of $v$ and $w$ is immaterial as long as they are adjacent.

Theorem 2.9 Let $G$ be a pleasant graph with $n$ vertices and let $v$ and $w$ be adjacent vertices in $G$. Then the bipolar coloring complex $\Delta_{G}(v, w)$ is a constructible complex. As a consequence, $\Delta_{G}(v, w)$ is homotopy-C M. In particular, $\Delta_{G}(v, w)$ is homotopy equivalent to a wedge of spheres of dimension $n-3$ with one sphere for each acyclic orientation $P$ of $G$ such that $\max P=\{v\}$ and $\min P=\{w\}$.

Proof: We use induction on the number of vertices and edges in $G$. The first base case is a graph with two edges $v w$ and $e$ (this graph clearly has two 2-connected components). We have already concluded that $\Delta_{\{e, v w\}}(v, w)$ is a cone over the first barycentric subdivision of the $(n-4)$-simplex and hence shellable. The second base case is the complete graph $K_{n}$ for $n=2$ and $n=3$; it is clear that $\Delta_{K_{n}}(v, w)$ is a sphere of dimension $n-3$ as desired.

Assume that $G$ is a pleasant graph with at least four vertices and three edges. Let $X$ be the 2-connected component in $G$ containing $\{v, w\}$.

First, suppose that $X=\{v, w\}$. The graph obtained by removing the edge $v w$ has the property that $v$ and $w$ belong to different connected (i.e., 1-connected) components. Namely, otherwise we would have a path from $v$ to $w$ in $G-v w$; the set $U$ of vertices in this path would have the property that $G(U)$ is 2-connected, being Hamiltonian.

Let $e$ be any edge in $G-v w$. We claim that $G-e$ and $G / e$ consist of at least two 2connected components. This is obvious for $G-e$; this graph contains at least one additional edge besides $v w$, and this edge is not contained in the 2-connected component containing $v$ and $w$, which is still $X$. Also, in $G / e$, the 2-connected component containing $v$ and $w$ remains equal to $X ; v$ and $w$ still belong to different connected components in $(G / e)-$ $v w=(G-v w) / e$. Finally, $G / e$ must contain at least two edges. Namely, by assumption, there is a third edge $f \neq e, v w$ in $G$, and this edge is identified with $v w$ in $G / e$ if and 
only if $\{e, f, v w\}$ forms a triangle $\{v x, w x, v w\}$. This would imply that $G(\{v, x, w\})$ is 2-connected, a contradiction to the maximality of $X$.

As a consequence, each of $G-e$ and $G / e$ is a pleasant graph. We may hence use induction, Lemmas 2.3, and 2.4 to conclude that $\Delta_{G}(v, w)$ is constructible.

Next, suppose that $X \supsetneqq\{v, w\}$. This means that $G(X)$ contains some edge $e \neq v w$. We claim that $G-e$ and $G / e$ are pleasant.

First, consider $G / e$. If $G$ is connected, then obviously $G / e$ is connected. Suppose that $G$ has at least two 2-connected components and let $S$ be the set of isolated vertices in $G$. By assumption, $H=G(V \backslash S)$ is not 2-connected. It is clear that $H / e$ does not contain any isolated vertices. Namely, at least one of the vertices in $e$ is adjacent to other vertices; they are both contained in $X$, which contains at least three elements. In particular, it suffices to show that there is a cutpoint in $H / e$. Let $x$ be a cutpoint in $H$. If $x \notin e$, then $(H / e)(V \backslash\{x\})=(H(V \backslash\{x\})) / e$, which immediately implies that $x$ remains a cutpoint in $H / e$. If $x \in e=x y$, then $H(V \backslash\{x, y\})$ is disconnected; $H(V \backslash\{x\})$ is disconnected with $y$ part of a connected component containing $X \backslash\{x\}$, which has size at least 2 . Yet, $H(V \backslash\{x, y\})=(H / e)((V \backslash\{y\}) \backslash\{x\})$, which implies that $x$ remains a cutpoint in $H / e$.

Next, consider $G-e$. It is clear that $G-e$ has at least as many 2-connected components as $G$. Namely, no 2-connected component in $G$ except $X$ contains $e$, which means that all 2-connected components except $X$ remain the same in $G-e$. Also, some subset of $X$ (containing at least $v$ and $w$ ) is a 2-connected component in $G$. In particular, $G-e$ contains at least two 2-connected components if $G$ does. Also, $G-e$ is connected if $G$ is connected; otherwise, $G(X \backslash\{x\})$ would be disconnected for at least one endpoint $x$ of $e$. As a consequence, $G-e$ is pleasant if $G$ is pleasant.

Since each of $G-e$ and $G / e$ is a pleasant graph, $\Delta_{G}(v, w)$ is constructible by induction, Lemmas 2.3, and 2.4.

For graphs without isolated vertices, the last statement in the Theorem is a consequence of the Greene-Zaslavsky Theorems 2.2 and 2.6. Other pleasant graphs are those with isolated vertices and at least two 2-connected components. By Theorem 2.8, such graphs (with or without isolated vertices) do not admit acyclic orientations with a unique source and a unique sink, which by Theorem 2.2 implies that $\chi_{G}^{\prime}(1)=0$. Theorem 2.6 yields the desired result.

The following result indicates that unpleasant graphs may not be so bad after all; $\Delta_{G}(v, w)$ turns out to be collapsible to a constructible subcomplex.

Theorem 2.10 Let $G$ be a nonempty graph and let $S$ be the set of isolated vertices in $G$. Let $v$ and $w$ be adjacent vertices in $G$. Then the bipolar coloring complex $\Delta_{G}(v, w)$ is collapsible to $\Delta_{G(V \backslash S)}(v, w)$. In particular, if $G$ is unpleasant, then $\Delta_{G}(v, w)$ is homotopy equivalent to a nonempty wedge of spheres of dimension $n-|S|-3$ with one sphere for each acyclic orientation $P$ of $G(V \backslash S)$ such that $\max P=\{v\}$ and $\min P=\{w\}$.

Proof: If $S$ is empty, then there is nothing to prove; note that $S$ is always nonempty whenever $G$ is unpleasant. Let $s \in S$. We want to find a collapse from $\Delta_{G}(v, w)$ to $\Delta_{0}=$ $\Delta_{G(V \backslash\{s\})}(v, w)$. Proceed in steps as follows. 
For a face $\mathcal{X}$ in $\Delta_{G}(v, w) \backslash \Delta_{0}$, let $T(\mathcal{X})$ be the smallest set in $\mathcal{X}$ containing $s$. Let $\left(T_{1}, T_{2}, \ldots, T_{r}\right)$ be a list containing all sets $T$ such that $T=T(\mathcal{X})$ for some face $\mathcal{X} \in$ $\Delta_{G}(v, w) \backslash \Delta_{0}$. Assume that the list is arranged such that $i \leq j$ whenever $T_{i} \supseteq T_{j}$. For $1 \leq j \leq r$, let $\Delta_{j}$ be the simplicial complex containing $\Delta_{0}$ and all faces $\mathcal{X}$ such that $T(\mathcal{X})=T_{i}$ for some $i \leq j$. Since $T(\mathcal{X}) \supseteq T\left(\mathcal{X}^{\prime}\right)$ whenever $\mathcal{X} \subseteq \mathcal{X}^{\prime}, \Delta_{j}$ is indeed a simplicial complex. Note that $\Delta_{r}=\Delta_{G}(v, w)$.

For $1 \leq j \leq r$, we collapse $\Delta_{j}$ to $\Delta_{j-1}$ as follows. $\mathcal{X}$ being a member of $\Delta_{j} \backslash \Delta_{j-1}$ means that $T_{j}$ is the smallest set in $\mathcal{X}$ containing $s$. Since $s$ is an isolated vertex, one may add or delete the set $T_{j} \backslash\{s\}$ without creating an element outside $\Delta_{j} \backslash \Delta_{j-1}$. Namely, $\mathcal{X} \cup\left\{T_{j} \backslash\{s\}\right\}$ remains a chain, as the largest set in $\mathcal{X}$ smaller than $T_{j}$ is a subset of $T_{j} \backslash\{s\}$. As a consequence, we can collapse $\Delta_{j}$ down to $\Delta_{j-1}$ using $T_{j} \backslash\{s\}$ as the "apex". In terms of discrete Morse theory [3], we may form a perfect matching on $\Delta_{j} \backslash \Delta_{j-1}$ by pairing $\mathcal{X} \backslash\left\{T_{j} \backslash\{s\}\right\}$ with $\mathcal{X} \cup\left\{T_{j} \backslash\{s\}\right\}$.

The last statement in the Theorem is a consequence of Theorem 2.9; the wedge of spheres is nonempty by Theorem 2.8 .

\section{Some positivity results}

In this section, we present some positivity results related to coloring complexes. We also give a topological interpretation of analogous positivity results by Linial [10] and Gessel [4].

For positive integers $l$ and $i$, it is well-known that there is a unique expansion $l=$ $\sum_{r=j}^{i}\left(\begin{array}{c}n_{r} \\ r\end{array}\right)$ such that $1 \leq j \leq n_{j}<\cdots<n_{i-1}<n_{i}$. Define

$$
l^{\langle i\rangle}=\sum_{r=j}^{i}\left(\begin{array}{c}
n_{r}+1 \\
r+1
\end{array}\right)
$$

$\left(0^{\langle i\rangle}=0\right)$. A sequence $\left(h_{0}, \ldots, h_{d}\right)$ is an $M$-vector if $h_{0}=1$ and $0 \leq h_{i+1} \leq h_{i}^{\langle i\rangle}$ for $1 \leq i \leq d-1$; see Stanley [16, Section 2.2]. We say that the corresponding polynomial $\sum_{i} h_{i} t^{i}$ forms an $M$-vector.

Let $R$ be a field or $\mathbb{Z}$. Let $\Delta$ and $\Sigma$ be simplicial complexes such that $\Delta \subseteq \Sigma$. The relative complex $\Sigma / \Delta$ is $C M / R$ if the reduced relative homology group $\tilde{H}_{i}\left(\operatorname{link}_{\Sigma}(\sigma), \operatorname{link}_{\Delta}(\sigma) ; R\right)$

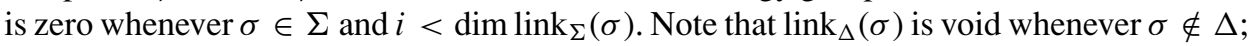
in this case, $\tilde{H}_{i}\left(\operatorname{link}_{\Sigma}(\sigma)\right.$, link $\left.\mathrm{H}_{\Delta}(\sigma) ; R\right)$ coincides with the ordinary reduced homology group $\tilde{H}_{i}\left(\operatorname{link}_{\Sigma}(\sigma) ; R\right)$. Define

$$
h(\Sigma / \Delta, t)=h(\Sigma, t)-(1-t)^{\operatorname{dim} \Sigma-\operatorname{dim} \Delta} \cdot h(\Delta, t) .
$$

The following two classical theorems are indispensable for this section; for proofs, see Stanley [16].

Theorem 3.1 If the simplicial complex $\Delta$ is $C M / R$, then the polynomial $h(\Delta, t)$ forms an $M$-vector. In particular, all coefficients in $h(\Delta, t)$ are nonnegative. 
Theorem 3.2 If $\Delta \subseteq \Sigma$ are simplicial complexes such that $\Sigma / \Delta$ is $C M / R$, then all coefficients in the polynomial $h(\Sigma / \Delta, t)$ are nonnegative.

A crucial observation in the proofs is that the Stanley-Reisner ring over $R$ of a $C M / R$ complex and the "face module" of a relative $C M / R$ complex are Cohen-Macaulay in the algebraic sense; see Reisner [13] and Stanley [15].

Lemma 3.3 (Stanley [16]) If $\Delta \subset \Sigma$ are $C M / R$ complexes and $\operatorname{dim} \Sigma-\operatorname{dim} \Delta \leq 1$, then $\Sigma / \Delta$ is $C M / R$.

Proof: Let $\sigma$ be a face in $\Sigma$. If $\sigma \notin \Delta$, then $\tilde{H}_{i}\left(\operatorname{link}_{\Sigma}(\sigma), \operatorname{link}_{\Delta}(\sigma)\right)=\tilde{H}_{i}\left(\operatorname{link}_{\Sigma}(\sigma)\right)$, which is zero whenever $i<\operatorname{dim}^{\operatorname{link}}(\sigma) ; \Sigma$ is $C M / R$. If $\sigma \in \Delta$ and $\operatorname{dim} \Sigma-\operatorname{dim} \Delta=1$, then, by the $C M / R$ property of $\Sigma$ and $\Delta$, the long exact sequence for $\left(\operatorname{link}_{\Sigma}(\sigma), \operatorname{link}_{\Delta}(\sigma)\right)$ (see Munkres [12]) vanishes except for the portion

$$
0 \rightarrow \tilde{H}_{d}\left(\operatorname{link}_{\Sigma}(\sigma)\right) \rightarrow \tilde{H}_{d}\left(\operatorname{link}_{\Sigma}(\sigma), \operatorname{link}_{\Delta}(\sigma)\right) \rightarrow \tilde{H}_{d-1}\left(\operatorname{link}_{\Delta}(\sigma)\right) \rightarrow 0
$$

$d=\operatorname{dim}_{\operatorname{link}_{\Sigma}}(\sigma)=\operatorname{dim}_{\operatorname{link}}(\sigma)+1$. If $\operatorname{dim} \Sigma=\operatorname{dim} \Delta$, then the long exact sequence vanishes except for the portion

$$
0 \rightarrow \tilde{H}_{d}\left(\operatorname{link}_{\Delta}(\sigma)\right) \rightarrow \tilde{H}_{d}\left(\operatorname{link}_{\Sigma}(\sigma)\right) \rightarrow \tilde{H}_{d}\left(\operatorname{link}_{\Sigma}(\sigma), \operatorname{link}_{\Delta}(\sigma)\right) \rightarrow 0 .
$$

In both cases, the consequence is that $\Sigma / \Delta$ is $C M / R$.

For the remainder of this section, let $G$ be a fixed nonempty graph and let $v$ and $w$ be fixed vertices in $G$. By Theorem 3.1, the following result is a consequence of the fact that the complexes $\Delta_{G}, \Delta_{G}(v)$, and $\Delta_{G}(v, w)$ are $C M / \mathbb{Z}$; see Theorems 1.4, 2.7, and 2.9.

Corollary 3.4 Let $G$ be a nonempty graph and let $v$ be a vertex in $G$. Then the polynomials $h\left(\Delta_{G}, t\right)$ and $h\left(\Delta_{G}(v), t\right)$ form $M$-vectors. If, in addition, $G$ is pleasant and $w$ is adjacent to $v$ in $G$, then the polynomial $h\left(\Delta_{G}(v, w), t\right)$ forms an $M$-vector. In particular, the given polynomials have nonnegative coefficients.

For a nonempty graph $G$, write

$$
\begin{aligned}
& A_{0}(G, t)=(1-t)^{n+1} \cdot \sum_{r \geq 0} \chi_{G}(r+1) t^{r} ; \\
& A_{1}(G, t)=(1-t)^{n} \cdot \sum_{r \geq 0} \frac{\chi_{G}(r+1)}{r+1} \cdot t^{r} \\
& A_{2}(G, t)=(1-t)^{n-1} \cdot \sum_{r \geq 1} \frac{\chi_{G}(r+1)}{r(r+1)} \cdot t^{r}
\end{aligned}
$$


Write $\Delta_{G}^{0}=\Delta_{G}, \Delta_{G}^{1}=\Delta_{G}(v)$, and $\Delta_{G}^{2}=\Delta_{G}(v, w)$. When examining $\Delta_{G}^{2}$, we will assume that $v w \in G$. By Theorems 1.6, 2.5, and 2.6, it is clear that

$$
A_{i}(G, t)=\frac{E_{n-i}(t)}{t}-(1-t) h\left(\Delta_{G}^{i}, t\right)
$$

for $0 \leq i \leq 2$ (for $i=2$, we assume that $G$ contains the edge $v w$ ); $E_{k}(t)$ is defined in (9). By Proposition 1.9, $E_{n}(t) / t, E_{n-1}(t) / t$, and $E_{n-2}(t) / t$ are the $h$-vectors of $\Sigma_{n}^{0}=\Sigma_{V}$, $\Sigma_{n}^{1}=\Sigma_{V}(v)$, and $\Sigma_{n}^{2}=\Sigma_{V}(v, w)$, respectively. By (16), this implies that

$$
A_{i}(G, t)=h\left(\Sigma_{n}^{i} / \Delta_{G}^{i}, t\right) .
$$

Namely, $\operatorname{dim} \Sigma_{n}^{i}=\operatorname{dim} \Delta_{G}^{i}+1$. This has the following interesting consequence.

Theorem 3.5 (Linial [10], Gessel [4]) For any graph $G$, all coefficients in $A_{0}(G, t)$ and $A_{1}(G, t)$ are nonnegative. In addition, if $G$ is pleasant, then all coefficients in $A_{2}(G, t)$ are nonnegative.

Remark Linial [10] was the first to prove the statement about $A_{0}(G, t)$, whereas the statement about $A_{2}(G, t)$ is due to Gessel [4]. Several authors have rediscovered Linial's result; see Gessel [4] and Steingrímsson [17] for references.

Proof: By Theorem 3.2 and (17), it suffices to prove that $\Sigma_{n}^{i} / \Delta_{G}^{i}$ is a relative $C M / \mathbb{Z}$ complex. Note that $\Sigma_{n}^{i}$ and $\Delta_{G}^{i}$ are $C M / \mathbb{Z}$ complexes in the usual sense; use Theorems 1.4, 2.7, and 2.9. Hence we are done by Lemma 3.3.

The following consequence of Theorem 3.5 and (16) puts a bound on how fast the sequence of coefficients in $h\left(\Delta_{G}^{i}, t\right)$ can increase.

Corollary 3.6 With assumptions for each $i$ as in Theorem 3.5,

$$
(1-t) h\left(\Delta_{G}^{i}, t\right) \leq \frac{E_{n-i}(t)}{t}
$$

the inequality holds coefficient-wise.

Finally, we show that $h\left(\Delta_{G}^{i}, t\right)$ is monotonely increasing in terms of $G$.

Proposition 3.7 Let $G$ be a graph and let $H$ be a proper subgraph of $G$. Then $h\left(\Delta_{H}^{i}, t\right) \leq$ $h\left(\Delta_{G}^{i}, t\right)$ for $0 \leq i \leq 2$ (for $i=2$, we assume that $G$ and $H$ are pleasant graphs containing the edge vw); the inequality holds coefficient-wise with strict inequality for at least one coefficient. 
Proof: For $H=G-e$, observe that

$$
\begin{aligned}
h\left(\Delta_{G}^{i}, t\right)-h\left(\Delta_{G-e}^{i}, t\right) & =\frac{-A_{i}(G, t)+A_{i}(G-e, t)}{1-t}=\frac{A_{i}(G / e, t)(1-t)}{1-t} \\
& =A_{i}(G / e, t)
\end{aligned}
$$

use (17) and (7). Indeed, it is easy to see that $\Delta_{G}^{i} / \Delta_{G-e}^{i}$ and $\Sigma_{n-1}^{i} / \Delta_{G / e}^{i}$ are isomorphic. Since $A_{i}(G / e, t)$ is a (nonzero) polynomial with nonnegative coefficients by Theorem 3.5, induction on $|G \backslash H|$ yields the desired result. An alternative approach would be to apply Theorems 3.2 and Lemma 3.3 to the relative complex $\Delta_{G}^{i} / \Delta_{H}^{i}$.

\section{Concluding remarks}

While we have been able to prove that the coloring complex and its polar subcomplexes are constructible, the problem of finding a shelling remains unsolved. We do believe that the complexes are shellable, but many "natural" candidates for shelling orders (e.g., different kinds of lexicographic order) turn out to fail in general.

We have considered three kinds of coloring complexes corresponding to the three polynomials $A_{0}(G, t), A_{1}(G, t)$, and $A_{2}(G, t)$ in Section 3 . Is it by any chance possible to define yet another coloring complex corresponding to a polynomial $A_{3}(G, t)$ defined in some natural manner? The most natural candidate for $A_{3}(G, t)$ is probably the polynomial obtained by replacing $\frac{\chi_{G}(r+1)}{r(r+1)}$ with $\frac{\chi_{G}(r+2)}{r(r+1)(r+2)}$ in the definition of $A_{2}(G, t)$ (this makes sense as soon as $G$ is not 3-colorable). Gessel [4] observed that there exists a connected graph $G$ such that some coefficients in $A_{3}(G, t)$ are negative.

\section{Acknowledgment}

I thank Einar Steingrímsson and Volkmar Welker for useful comments and fruitful discussions. The problem of determining whether the coloring complex is Cohen-Macaulay was suggested by Einar Steingrímsson. Volkmar Welker identified the coloring complex as the complex $\Delta_{m, J}$ of Herzog et al. [7].

This work was carried out at Fachbereich Mathematik und Informatik, PhilippsUniversität Marburg.

\section{References}

1. A. Björner, "Topological methods," in Handbook of Combinatorics, R. Graham, M. Grötschel, and L. Lovász (Eds.), North-Holland/Elsevier, Amsterdam, 1995, pp. 1819-1872.

2. A. Björner and M. Wachs, "On lexicographically shellable posets," Trans. Amer. Math. Soc. 277 (1983), 323-341.

3. R. Forman, "Morse theory for cell complexes," Adv. Math. 134 (1998), 90-145.

4. I.M. Gessel, "Acyclic orientations and chromatic generating functions," Discrete Math. 232 (2001), 119-130.

5. D. Gebhard and B. Sagan, "Sinks in acyclic orientations of graphs," J. Combin. Theory, Ser. B, 80 (2000) $130-146$. 
6. C. Greene and T. Zaslavsky, "On the interpretation of Whitney numbers through arrangements of hyperplanes, zonotopes, non-radon partitions, and orientations of graphs," Trans. Amer. Math. Soc. 280 (1983), 97-126.

7. J. Herzog, V. Reiner, and V. Welker, “The Koszul property in affine semigroup rings,” Pacific J. Math. 186 (1998), 39-65.

8. M. Hochster, "Rings of invariants of tori, Cohen-Macaulay rings generated by monomials, and polytopes," Ann. Math. 96 (1972), 318-337.

9. A. Lempel, S. Even, and I. Cederbaum, "An algorithm for planarity testing of graphs," Theory of Graphs, Gordon and Breach (Eds.), 1967, pp. 215-232.

10. N. Linial, "Graph coloring and monotone functions on posets," Discrete Math. 58 (1986), 97-98.

11. L. Lovász, Combinatorial Problems and Exercises, 2nd edition, North-Holland, Amsterdam, 1993.

12. J.R. Munkres, Elements of Algebraic Topology, Menlo Park, CA, Addison-Wesley, 1984.

13. G. Reisner, "Cohen-Macaulay quotients of polynomial rings," Adv. Math. 21 (1976), 30-49.

14. R.P. Stanley, “Acyclic orientations of graphs," Discrete Math. 5 (1973), 171-178.

15. R.P. Stanley, "Generalized h-vectors, intersection cohomology of toric varieties, and related results," in Commutative Algebra and Combinatorics, M. Nagata and H. Matsumura (Eds.), Advanced Studies in Pure Mathematics 11, Kinokuniya, Tokyo, and North-Holland, Amsterdam/New York, 1987, pp. 187-213.

16. R.P. Stanley, Combinatorics and Commutative Algebra, 2nd edition, Progress in Mathematics, vol. 41, Birkhäuser, Boston/Basel/Stuttgart, 1996.

17. E. Steingrímsson, "The coloring ideal and coloring complex of a graph,” J. Alg. Comb. 14 (2001), 73-84. 